\title{
Author Correction: The microscopic role of deformation in the dynamics of soft colloids
}

Nicoletta Gnan (10) and Emanuela Zaccarelli (1)

Correction to: Nature Physics https://doi.org/10.1038/s41567-019-0480-1, published online 1 April 2019.

The original version of this Article implied that the model reported described a system in equilibrium. Based on analysis of subsequent simulations, we have determined that our assumption that the model describes an equilibrium system was incorrect. Thus, the sentence "However, no evidence has been provided so far of compressed exponential relaxation in a microscopic model undergoing spontaneous relaxation (in equilibrium), such as the present one." should have read "However, no evidence has been provided so far of compressed exponential relaxation in a microscopic elastic model undergoing spontaneous relaxation." All other results are unaffected. Furthermore, in equation (3) all instances of ' $r / \sigma_{\mathrm{H}}$ ' should have been ' $2 r / \sigma_{\mathrm{H}}$ '; the correct equation is:

$$
V_{H}(r)=U\left(1-2 r / \sigma_{H}\right)^{5 / 2} \Theta\left(1-2 r / \sigma_{H}\right)
$$

The online versions of the Article have been amended.

Published online: 19 May 2021

https://doi.org/10.1038/s41567-021-01252-w

(c) The Author(s), under exclusive licence to Springer Nature Limited 2021

\section{Author Correction: Generalized superradiance for producing broadband coherent radiation with transversely modulated arbitrarily diluted bunches}

\section{J. Vieira (D), M. Pardal, J. T. Mendonça and R. A. Fonseca (D)}

Correction to: Nature Physics https://doi.org/10.1038/s41567-020-0995-5, published online 14 September 2020.

In the version of this Article originally published, in the Acknowledgements, the support from the European Union's Horizon 2020 research and innovation programme under grant agreement no. 871124 (Laserlab-Europe) was mistakenly not mentioned; this information has now been added to the sentence beginning "This work was partially supported by.... The online versions of the Article have been amended.

Published online: 17 May 2021

https://doi.org/10.1038/s41567-021-01257-5

(C) The Author(s), under exclusive licence to Springer Nature Limited 2021 